\title{
Development of Multiplex Allele-specific PCR Assay for BRAFV600E Mutation Detection in Human Cancer Cells
}

\author{
Young Doo Kim \\ St. Johnsbury Academy Jeju, 1159, Gueok-ri, Daejeong-eup, Seogwipo-si,Jeju-do, Republic of Korea; gosyber@suwon.ac.kr
}

ABSTRACT: BRAF mutation is commonly found in many types of human cancers. To detect the BRAFV600E mutation in cancer cells, a low-cost detection method was developed using multiplex allele-specific PCR assay. This method was designed to amplify three different size products in a single reaction: a 403-base pair (bp) for PCR amplification control, a 300 bp for wildtype (wt) allele-specific product, a $151 \mathrm{bp}$ for BRAFV600E allele-specific product. The method was further validated in three different cancer cell lines with or without the BRAFV600E mutation: A172 (BARFwt), SK-MEL2 (BRAFwt), and A375SM (BARFwt/V600E), which was confirmed by Sanger sequencing. After genomic DNA was extracted from all three cell lines, an allele-specific PCR assay was performed. Genomic DNA from A172 and SK-MEL2 amplified only 403 bp (PCR amplification control) and $300 \mathrm{bp}$ (BRAFwt) products, but not $151 \mathrm{bp}$ (BRAFV600E) products. However, genomic DNA from A375SM successfully amplified all three sizes of products, including $151 \mathrm{bp}$ (BRAFV600E), indicating that A375SM harbors BRAFwt/ V600E heterozygous mutation. These results indicate that this novel multiplex allele-specific PCR is sensitive enough to detect BRAFV600E harboring cancer cells. Overall, this method not only provides a new tool for the rapid detection of the BRAFV600E mutation but also can be used for early detection of point mutations of various biomarker genes.

KEYWORDS: Molecular Biology; Cancer; Melanoma; BRAF mutation.

\section{- Introduction}

Among many genetic diseases, this study focuses on detecting the BRAFV600E mutations in human cancers. BRAF mutations occur in about $8 \%$ of all human cancers. ${ }^{1}$ Especially, $80 \%$ of melanoma harbor BRAFV600E mutation ${ }^{2}$ and over $90 \%$ of these are a single nucleotide substitution of glutamic acid for valine. ${ }^{3}$ BRAF gene encodes a kinase protein, which can activate the mitogen activated protein kinase (MAPK) pathway. This signal can be activated by epidermal growth factor (EGF) through binding to the EGFR receptor. ${ }^{4}$ The mutations in BRAF allows constitutive MAPK pathway to mediate the cellular growth and metastasis in cancer cells. ${ }^{5}$ Furthermore, BRAFV600E has been known to be involved in melanoma progression through various mechanisms. Such mechanisms include, evasion of apoptosis, unchecked proliferation, angiogenesis, metastasis, and evasion of immune response (Figure 1). ${ }^{6}$ In order to detect this mutation as early as possible, a sensitive, specific, and low-cost assay needs to be developed. According to a market trend analysis report, the market for cancer diagnostic is increasing rapidly at an annual growth rate of $42 \% .{ }^{6,7}$ and of different methods, polymerase chain reaction (PCR) is known as one of the most reliable and efficient ways to detect cancer at early stages. ${ }^{7}$ This study is, therefore, focused on developing an accurate and efficient method for detecting BRAFV600E mutation using PCR method.

\section{- Methods}

\section{Cell culture and maintenance:}

Three human cancer cell lines, A172 (brain), SK-MEL2 (skin), and A375SM (skin), were obtained from the Korean Cell Line Bank (Seoul, Korea). Gibco RPMI-1640 medium supplemented with $10 \%$ FBS (Thermo Fisher) and $1 \%$ penicillin and streptomycin were used. The cells were kept at $37^{\circ} \mathrm{C}$ in a $\mathrm{CO} 2$ incubator.
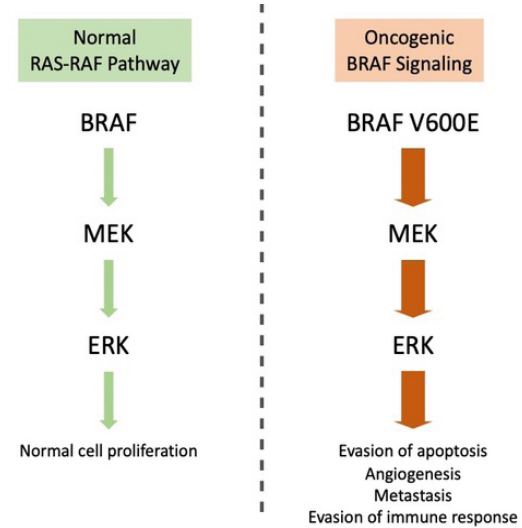

Figure 1: Comparison of RAS-RAF pathways in normal and BRAF mutation-derived oncogenic conditions. Mutation in BRAFV600E leads to upregulation of MEK-ERK pathway, which in turn, triggers various oncogenic conditions.

\section{DNA extraction:}

After human cancer cell lines were harvested, DNA was purified by QIAamp DNA Mini kit (Qiagen). Firstly, the cancer cells were suspended with $200 \mu \mathrm{L}$ PBS. Then, $200 \mu \mathrm{L}$ buffer AL was added. After the samples were incubated at $56^{\circ} \mathrm{C}$ for $10 \mathrm{~min}, 200 \mu \mathrm{L}$ ethanol was added. Then, the samples were moved to AIAamp Mini spin column. After the centrifugation was applied for $1 \mathrm{~min}$, the flow-through solutions were removed. Then, the column was washed with 500 $\mu \mathrm{L}$ buffer AW1 and $500 \mu \mathrm{L}$ buffer AW2. Finally, the purified DNA was eluted with $200 \mu \mathrm{L}$ AE buffer. 


\section{Allele-specific PCR:}

The primer sequences are indicated in Figure 2. PCR was performed in a $20 \mu \mathrm{L}$ final volume containing $1 \times$ buffer, 2 $\mathrm{mM} \mathrm{MgCl2}$, Taq DNA polymerase (Bioneer), $250 \mathrm{uM}$ of each dNTP, 1 pmol of each primer, and $100 \mathrm{ng}$ genomic DNA template. The following condition was used for PCR amplification: $95^{\circ} \mathrm{C}$ for $5 \mathrm{~min}, 35$ cycles of $95^{\circ} \mathrm{C}$ for $20 \mathrm{sec}$, $56 \mathrm{oC}$ for $30 \mathrm{sec}$, and $72{ }^{\circ} \mathrm{C}$ for $30 \mathrm{sec}$ with a final extension at $72{ }^{\circ} \mathrm{C}$ for $5 \mathrm{~min}$ as shown in Figure 1.

\section{Agarose gel electrophoresis:}

PCR products were analyzed by $2 \%$ agarose gel electrophoresis. The gels were run at $100 \mathrm{~V}$ for 30 minutes. Red-safe (intron) was used to stain the DNA products. Blue light illuminator (B-box) was used to visualize the DNA products.

\section{CSanger Sequencing:}

1 microliter of the genomic DNA samples were subjected to PCR amplification. The changes in the genomic DNA sequences of the cancer cells were analyzed by Sanger sequencing analysis (Bioneer, Korea) of a PCR fragment that was amplified from the BRAF gene.

\section{Results and Discussion}

BRAF gene
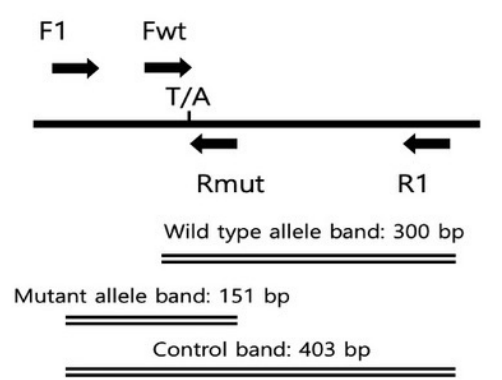

\section{F1: 5'- ACTCTTCATAATGCTTGCTCTGATAGG -3' \\ R1: 5'- TGTTTGAAATACACTGAAACTGGT -3' \\ Fwt: 5'- GGTGATTTTGGTCTAGCTACAGT -3' \\ Rmt: 5'- ATGGGACCCACTCCATCGAGATTTCT -3'}

Figure 2: Primer design of multiplex BRAFV600E allele-specific PCR. Forward Primer 1 (F1) and Reverse primer 1 (R1) generates the control band (403 bp). Forward primer for wild type ( $\mathrm{Fwt}$ ) and R1 generates the wild type allele band (300 bp). F1 and Reverse primer for mutation (Rmt) generates the mutant allele band ( $151 \mathrm{bp})$. The wild type sequence is $\mathrm{T}$ and the mutant sequence is $\mathrm{A}$. The position of the mutation is indicated as T/A in the figure.

The multiplex BRAFV600E allele-specific PCR contained 4 primers in a single PCR reaction tube (Figure 2). Two external primers, F1 and R1, were designed to amplify the control fragment of $403 \mathrm{bp}$, spanning through the entire region including the wild type and mutant allele bands. Two internal primers, Rmut and Fwt, were designed with either specificity for the mutant sequence or the wild-type sequences, respectively. The mutant and wild-type allele bands were differentiated by the size of the fragment so that the bands would be visibly distinguishable in agarose gel electrophoresis analysis: the amplified wild type fragment was $300 \mathrm{bp}$ and amplified mutant fragment was $151 \mathrm{bp}$ (Figure 2). The optimal PCR condition was determined by varying the parameters, such as annealing temperature and PCR cycle numbers, through multiple experiments.

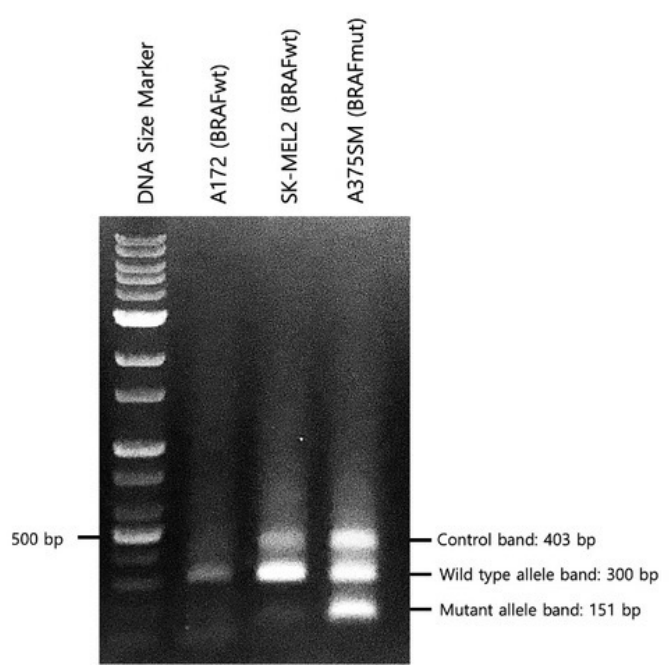

Figure 3: Specificity of BRAFV600E allele-specific PCR was analyzed using three different human cancer cell lines: A172 (BRAFwt), SK-MEL2 (BRAFwt), and A375SM (BRAFV600E). A172 and SK-MEL2 contains wild type allele and A375SM contains both wild type and mutant allele band.

To assess the analytical specificity of the assay, one BRAFV600E mutation containing cell line (A375SM) and two BRAF wild type containing cell lines (A172 and SKMEL2) were used in this multiplex PCR assay. $403 \mathrm{bp}$ of control bands and $300 \mathrm{bp}$ wild type allele bands were detected in all three cell lines. A $151 \mathrm{bp}$ mutant allele band was detected in only A375SM suggesting that A375SM had a heterozygous mutation (BRAFwt/V600E) (Figure 3). Also, the size of the products can be clearly separated, and no amplified bands overlapped in the agarose gel. A higher percentage of agarose gel and low molecular weight DNA marker will enhance the resolution.

When the DNA sequence of each cell line was also validated with Sanger sequencing, the results were identical to our BRAFV600E allele-specific PCR result (Figure 4), in-

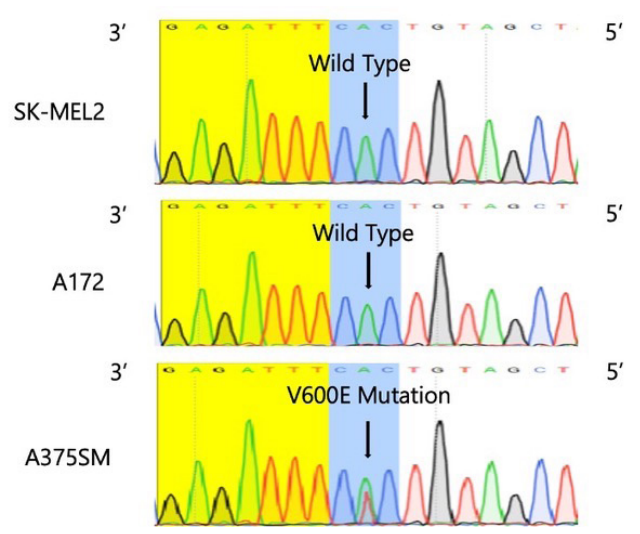

Figure 4: Sanger sequencing result showing wild type and BRAFV600E DNA sequence from SK-MEL2, A172, and A375SM cell lines. The reverse complement sequence is indicated in this figure. The reverse complement sequence of CAC is GTG, which encodes valine $(\mathrm{V})$ on SK-MEL2 and A172. The reverse complement sequence of CTC is GAG, which encodes glutamic acid (E). This result indicates SK-MEL2 and A172 has homozygous wild type allele. A375SM sequence shows that both type of nucleic acids, A and T, were shown at the position of V600E. This result indicates that $\mathrm{A} 375 \mathrm{SM}$ has a $\mathrm{V} 600 \mathrm{E}$ heterozygous mutation: contains a mutation of one allele and a wild type of another allele. 


\section{Conclusions}

Even though BRAFV600E mutation testing is widely used in various types of cancer patient samples, the previous method was very limited by its high cost and requirement of expensive lab equipment. ${ }^{8}$ Therefore, the need for simple BRAF mutation testing is increasing to apply for genetic testing and chemotherapy drug development. To develop a fast and specific detection of BRAFV600E mutation in many types of cancer cells, a multiplex PCR reaction was designed. After performing hundreds of $\mathrm{PCR}$ reaction, the primer and PCR conditions were finally optimized. Also, many different concentrations of primers were tested to increase the specificity. Finally, the most optimized condition was discovered. This sensitive, low-cost, and fast PCR assay amplifies a control fragment of DNA and serves as a positive control for PCR reaction and DNA quality. When the PCR product was analyzed, three different size products were detected in agarose gels.

In this study, the DNA extracted from only three different human cancer cell lines was used to detect BRAFV600E. Therefore, more cancer cells with BRAFV600E should be tested in future to confirm this method. In addition, this novel assay can be easily performed by many basic molecular laboratories. This method can be potentially applied to detect many other point mutations found in cancer tissues and genetic disease. Also, it is applicable to detect the cancer cells with BRAFV600E in the peripheral blood.

\section{Acknowledgements}

I would like to thank Dr. Woo Rin Lee from University of Suwon for his advice and support for this research.

\section{- References}

1. Dong, J.; Phelps, R. G.; Qiao, R.; Yao, S.; Benard, O.; Ronai, Z.; Aaronson, S. A., BRAF oncogenic mutations correlate with ' progression rather than initiation of human melanoma. Cancer research 2003, 63 (14), 3883-3885.

2. Menzies, A. M.; Lum, T.; Wilmott, J. S.; Hyman, J.; Kefford, R. F.; Thompson, J. F.; O’Toole, S.; Long, G. V.; Scolyer, R. A., Intrapatient homogeneity of BRAFV600E expression in melanoma. The American journal of surgical pathology 2014, 38 (3), 377-382.

3. Ascierto, P. A.; Kirkwood, J.M.; Grob, J.-J.; Simeone, E.; Grimaldi, A. M.; Maio, M.; Palmieri, G.; Testori, A.; Marincola, F. M.; Mozzillo, N., The role of BRAF V600 mutation in melanoma. Journal of Translational Medicine 2012, 10 (1), 85.

4. Sumimoto, H.; Imabayashi, F.; Iwata, T.; Kawakami, Y., The BRAF-MAPK signaling pathway is essential for cancer-immune evasion in human melanoma cells. The Journal of experimental medicine 2006, 203 (7), 1651-1656.

5. Tian, Y.; Guo, W., A review of the molecular pathways involved in resistance to BRAF inhibitors in patients with advanced-stage melanoma. Medical science monitor: international medical journal of experimental and clinical research 2020, 26, e920957-1.

6. Maurer, G.; Tarkowski, B.; Baccarini, M., Raf kinases in cancerroles and therapeutic opportunities. Oncogene 2011, 30 (32), 3477-3488.

7. Seo, J. H.; Lee, J. W.; Cho, D., The market trend analysis and prospects of cancer molecular diagnostics kits. Biomaterials Research 2018, 22 (1), 2.

8.Tan, Y. H.; Liu, Y.; Eu, K. W.; Ang, P. W.; Li, W. Q.; Tellez, M. S.; Iacopetta, B.; Soong, R., Detection of BRAF V600E mutation by pyrosequencing. Pathology 2008, 40 (3), 295-298.

\section{Author}

Young Doo is currently attending Saint Johnsbury Academy in Jeju. He is interested in studying biology and plans to major in molecular biology in university. He is interested in the function of genetic material or genomes in organisms and wants to explore it more deeply. 